\title{
Parathyroid hormone reserve in 22q11.2 deletion syndrome
}

Chirag R. Kapadia, MD ${ }^{1,2}$, Yuran E. Kim, BS ${ }^{1}$, Donna M. McDonald-McGinn, MS, CGC ${ }^{3}$, Elaine H. Zackai, MD ${ }^{2,3,4}$, and Lorraine E. Levitt Katz, $M D^{1,2}$

\begin{abstract}
Objective: We hypothesized that most patients with 22q11.2 deletion and a history of hypocalcemia have inadequate parathyroid function, manifested by intact parathyroid hormone levels below normal. We aimed to evaluate intact parathyroid hormone levels both during normocalcemia and at hypocalcemia, in this population. Study Design: Retrospective chart review of 103 patients with 22q11.2 deletion born since 1997 and cared for at the Children's Hospital of Philadelphia. Calcium and intact parathyroid hormone drawn simultaneously were recorded, along with clinical presentation at hypocalcemia. Results: Forty-seven simultaneous Ca/intact parathyroid hormone values were available. Seventy-nine percent of calcium levels and $81 \%$ of parathyroid hormone levels were within normal range. There were 19 patients with a history of symptomatic hypocalcemia, for whom any available simultaneous $\mathrm{Ca}$ /parathyroid hormone levels, before, during, or after hypocalcemia were analyzed. In this subgroup, $59 \%$ of calcium and $76 \%$ of parathyroid hormone levels were normal. None had an intact parathyroid hormone of $>39.2 \mathrm{pg} / \mathrm{mL}$ at hypocalcemia. Seventy-three percent of hypocalcemic events had a precipitating stressor. Conclusions: Hypoparathyroidism in 22q11.2 deletion is mild, manifesting as a phenomenon of decreased parathyroid hormone reserve. Subjects are normocalcemic most of the time, but are unable to mount elevated intact parathyroid hormone levels, and therefore unable to correct hypocalcemia, in response to stressors. Genet Med 2008:10(3):224-228.
\end{abstract}

Key Words: parathyroid hormone, PTH, calcium, Digeorge, 22q11.2, hypocalcemia, hypoparathyroidism, intact PTH

Microdeletion of chromosome 22q11 occurs with an incidence of approximately $1 / 4000-7000$ live births. ${ }^{1-3}$ This deletion results in failure of development of the derivatives of the 3rd and 4th pharyngeal pouches, with anomalous migration of the cells derived from the cephalic neural crest. ${ }^{4}$ Clinical expression varies from one patient to another. Features can include congenital cardiac defects, hypocalcemia, short stature, immunodeficiency from thymic hypoplasia, palate abnormalities, cognitive impairment, and minor facial dysmorphism. ${ }^{5}$

Hypocalcemia due to hypoparathyroidism in this population was described originally by Digeorge. ${ }^{6}$ The parathyroids are derivatives of the $3 \mathrm{rd}$ and 4 th pharyngeal pouches, and therefore hypoparathyroidism in this syndrome is not unexpected. It has been documented by aplasia or hypoplasia of parathyroid glands at surgery or autopsy. ${ }^{7}$ Our center previously reported hypocalcemia in 77 of 158 (49\%) patients. ${ }^{8,9}$

\footnotetext{
From the ${ }^{1}$ Division of Endocrinology, Children's Hospital of Philadelphia; ${ }^{2}$ Department of Pediatrics, University of Pennsylvania; ${ }^{3}$ Division of Human Genetics and Molecular Biology, Children's Hospital of Philadelphia; and ${ }^{4}$ Division of Obstetrics and Gynecology, Hospital of the University of Pennsylvania, Philadelphia, Pennsylvania.

Chirag Kapadia, MD, Endocrinology, 34th Street and Civic Center Boulevard, Philadelphia, PA 19104.E-mail:kapadiac@email.chop.edu.

Disclosure: The authors declare no conflicts of interest.

Submitted for publication August 30, 2007.

Accepted for publication October 17, 2007.

DOI: 10.1097/GIM.0b013e3181634edf
}

Brauner et al. ${ }^{10}$ combined a cross-sectional study with historical review to reveal abnormal parathyroid gland function in $69 \%$ of patients. However, hypoparathyroidism in 22q11.2 deletion syndrome has been difficult to characterize. The varying degrees of parathyroid dysfunction in the 22q11.2 deletion population, and even intra-individual variation in parathyroid function, have been described by multiple authors. ${ }^{1,5,10-15}$

We hypothesized that most patients with the syndrome and a history of hypocalcemia have inadequate parathyroid function, which would be manifested by intact parathyroid hormone (PTH) levels below the lower limit of normal. The aims of our study were (1) to determine the prevalence of both neonatal and later-occurring hypocalcemia in a large hospitalbased population, (2) to evaluate PTH levels during normocalcemia, and at the time of hypocalcemia, in subjects with 22q11.2 deletion, and (3) to define parathyroid function in patients with a known history of hypocalcemia.

\section{METHODS AND ANALYSIS}

An institutional review board-approved database of patients with 22q11.2 deletion was obtained. Subjects with a date of birth earlier than 1997 were excluded because of difficulty in searching records before this date (after this date, a computerbased system was available). There were 103 patients, born 
Relationship of calcium and intact PTH in 22q11.2 deletion syndrome

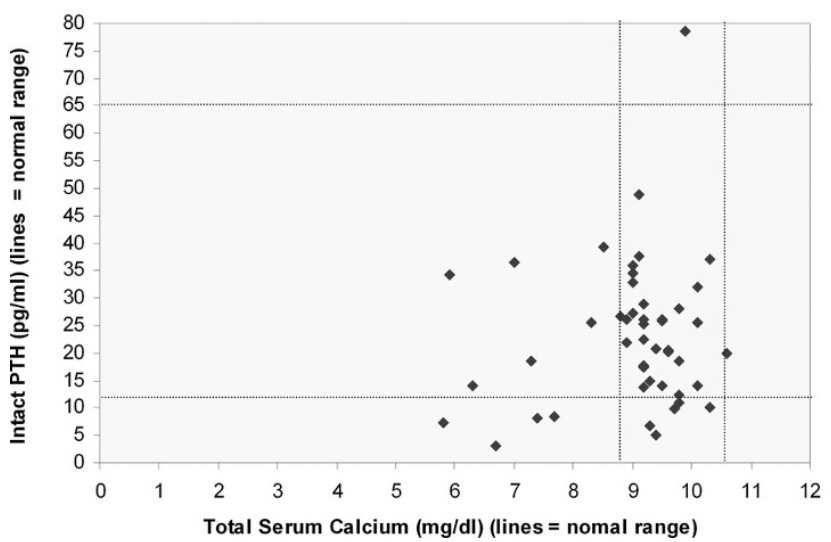

Fig. 1. Scatterplot of 47 simultaneous $P T H$ and calcium values obtained from all patients in study. Lines represent pediatric normal range.

between 1997 and July 2005, with confirmed 22q11.2 deletion, and these patients served as the sample for the study. Records were reviewed for date of genetic diagnosis. The records were then reviewed for any serum calcium drawn simultaneously with an intact PTH. For the purposes of the study, only PTH values drawn concurrently with serum total calcium were accepted. The data were logged into a database, and were then analyzed for the parameters described in the Results below. The pediatric normal range for calcium was defined as $8.8-$ $10.6 \mathrm{mg} / \mathrm{dL}$, and the pediatric normal range for PTH was defined as $12-65 \mathrm{pg} / \mathrm{mL}$, as these are the normal ranges used at the Children's Hospital of Philadelphia laboratory, where assays were run (Immulite 2000, DPC, Los Angeles, CA).

Simultaneous calcium and PTH values were available for 33 patients. Eight patients had more than 1 set of simultaneous calcium and PTH values. To ensure that we were capturing discrete events, values were included in analysis only if they occurred at least 1 month apart. If values occurred within 1 month of each other, the first value only was used for analysis. The total number of concurrent calcium/PTH values was 47 , as several patients had more than 1 set of concurrent calcium/ PTH values drawn at least 1 month apart. The database was narrowed to the subset of subjects with a documented, defined history of hypocalcemia, and any set of simultaneous calcium and PTH values available for these patients, whether drawn before, after, or during the hypocalcemic episode. Lastly, clinical data from the subgroup of subjects with an intact PTH value drawn specifically at the time of hypocalcemia were examined; there were eight such patients.

\section{RESULTS}

Date of genetic diagnosis was available for 76 of the patients. Of these, $47(62 \%)$ were diagnosed within the first month of life, 18 (24\%) were diagnosed between 1 month and 1 year of life, and 11 (14\%) were diagnosed after 1 year of life.

Hypocalcemia at any point in time, either evident through low serum calcium values or clearly documented in the Endocrine chart, was noted in 57 of 103 patients (55\%). Neonatal hypocalcemia (defined as occurring within the first month) was noted in 37 of 103 patients (36\%). At least 14 patients, or $14 \%$ of the patient pool, had hypocalcemia occurring for the first time after 1 month of age. Of these 14, 6 patients had the initial hypocalcemia event between 1 and 3 months of age; the initial presentation of the other 8 ( $8 \%$ of the population) ranged between 29 months and 7 years of age.

Figure 1 is a scatterplot of all 47 simultaneous PTH and calcium values that were obtained in the 103 patients whose data were examined. The lines on Figure 1 represent pediatric normal ranges for serum calcium and intact PTH. Thirtyseven out of the 47 calcium values (79\%) were within the normal range, as were 38 of $47 \mathrm{PTH}$ values (81\%). Only 10 of 46 calcium values and 8 of $47 \mathrm{PTH}$ values were below the normal range.

Figure 2 is a scatterplot of values obtained from the $19 \mathrm{pa}-$ tients with a history of hypocalcemia, who had simultaneous PTH and calcium values drawn at some point. Twenty-nine simultaneous calcium/intact PTH values were available from these 19 patients. Twelve of the values reflect hypocalcemia at the time of the draw, but 17 are normocalcemic. Six of the values $(21 \%)$ reflect classic hypoparathyroidism (with a PTH $<12$

\section{Simultaneous calcium and PTH values in $22 q 11$ deletion patients with a documented history of hypocalcemia}

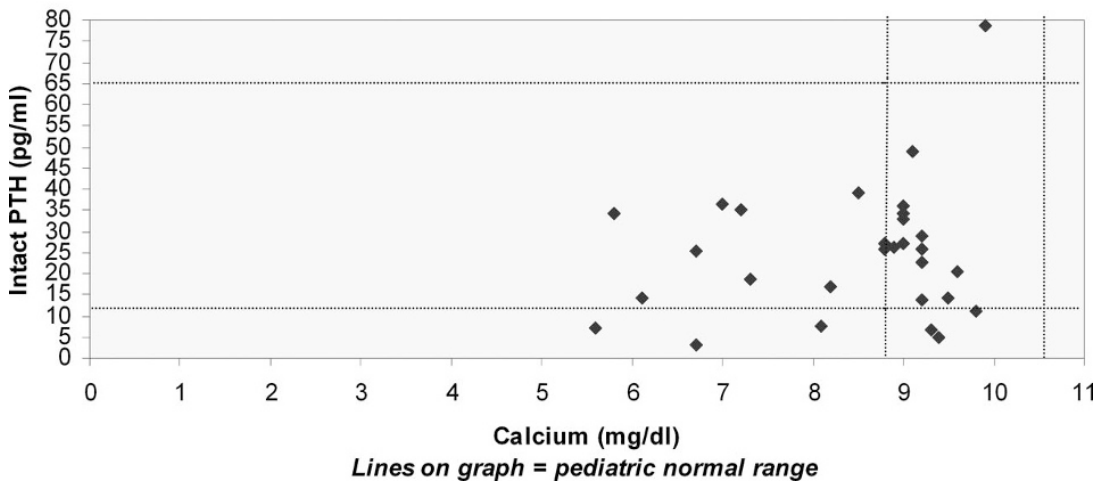

Fig. 2. Scatterplot of 29 simultaneous PTH and Calcium values obtained in 19 patients with a documented history of symptomatic hypocalcemia. Lines represent pediatric normal range. 
Table 1

Clinical characteristics of patients with intact PTH values drawn simultaneously with a serum calcium level during a hypocalcemic episode

\begin{tabular}{|c|c|c|c|c|c|c|c|c|}
\hline Patient & Sex & Phenotype & $\begin{array}{l}\text { Age of first } \\
\text { low Ca }\end{array}$ & $\begin{array}{l}\text { Clinical status at time } \\
\text { of first low Ca }\end{array}$ & $\begin{array}{l}\text { Serum Ca at } \\
\text { first low Ca }\end{array}$ & $\begin{array}{l}\text { Intact PTH } \\
\text { level }\end{array}$ & $\begin{array}{l}\text { Age of recurrence of } \\
\text { hypocalcemia }\end{array}$ & $\begin{array}{l}\text { Clinical status at time } \\
\text { of recurrence }\end{array}$ \\
\hline 1 & $\mathrm{~F}$ & VSD & 19 days & $\begin{array}{l}\text { Fever, rule-out sepsis } \\
\text { hospitalization }\end{array}$ & 5.9 & 34.2 & $2 \mathrm{mo}$ & VSD repair \\
\hline 2 & $\mathrm{~F}$ & $\begin{array}{l}\text { Earfolds, lymphopenia, } \\
\text { TA, VSD }\end{array}$ & 2 days & Postoperative & 7.4 & 8.1 & $5 \mathrm{wk}$ & Twitching \\
\hline 3 & M & TA, VSD & 7 days & Postoperative & 8.5 & 39.2 & $6 \mathrm{mo}$ & Not known \\
\hline 4 & $\mathrm{~F}$ & Hypocalcemic seizures & 6 days & Seizures & 7.3 & 18.6 & None & NA \\
\hline 5 & M & TOF & 1 day & Postdelivery & 7.0 & 36.5 & $5 \mathrm{mo}$ & Postoperative for TOF \\
\hline 6 & $\mathrm{M}$ & $\begin{array}{l}\text { Hypocalcemic seizures, } \\
\text { TOF, VSD }\end{array}$ & 13 days & Seizures & 6.1 & 14.1 & $2 \mathrm{mo}, 10 \mathrm{mo}$ & $\begin{array}{l}\text { Fevers, pneumonia, } \\
\text { hospitalization }\end{array}$ \\
\hline 7 & $\mathrm{~F}$ & TOF & 1 day & Postdelivery & 7.1 & 7.3 & $3 \mathrm{mo}, 14 \mathrm{mo}$ & $\begin{array}{l}\text { Postoperative for TOF; } \\
\text { second recurrence } \\
\text { with malrotation }\end{array}$ \\
\hline 8 & $\mathrm{~F}$ & TA, failure to thrive & $3 \mathrm{yr}$ & Unknown & 6.7 & 3.1 & None & NA \\
\hline
\end{tabular}

Ca, calcium; VSD, ventricular septal defect; TA, truncus arteriosus; TOF, tetralogy of Fallot; NA, not available.

$\mathrm{pg} / \mathrm{mL}$ ); however, $22 \mathrm{PTH}$ values (76\%) fell within the reference normal range of $12-65 \mathrm{pg} / \mathrm{mL}$ ( 1 value was elevated).

There were eight patients with a low calcium value drawn simultaneously with a PTH value. Data from these eight subjects are shown in Table 1. Three of these eight subjects had a PTH value below $12 \mathrm{pg} / \mathrm{mL}$ (the lower limit of the normal range) at the time of the hypocalcemia. The mean intact PTH at the time of hypocalcemia was $20.1 \mathrm{pg} / \mathrm{mL}$. None of the subjects had an intact PTH level $>39.2 \mathrm{pg} / \mathrm{mL}$ at the time of hypocalcemia. Of the 16 distinct hypocalcemic events that occurred in these eight patients, four were postoperative, two were within 1 day of birth (postdelivery), and five were associated with illness. Thus, 11 of 15 (73\%) of hypocalcemic events had a precipitating stressor. Six of the eight patients had more than one episode of hypocalcemia.

\section{DISCUSSION}

We have demonstrated that hypoparathyroidism in 22q11.2 deletion is a mild variant of the disease. Hypoparathyroidism in this syndrome generally seems to manifest in most cases as a phenomenon of decreased PTH reserve, with a predisposition to hypocalcemia during periods of stress or illness.

A key finding demonstrated by these data are that at the time of hypocalcemia, intact PTH levels in this population are generally within the normal range. In five of eight subjects, hypocalcemia was accompanied by an intact PTH within the low to mid-normal range; therefore, an intact PTH in this range cannot be taken as evidence against the diagnosis of hypoparathyroidism. This has been a source of confusion for clinicians, and has even been reported as "pseudohypoparathyroidism" in the literature. ${ }^{8}$

However, hypoparathyroid subjects with 22q11.2 deletion are unable to mount elevated PTH levels, and therefore unable to fully correct hypocalcemia, in response to stressors. This is illustrated in Figures 1 and 2, along with Table 1, showing that none of the intact PTH levels at hypocalcemia extends into an elevated or high-normal range. None of the subjects generated an intact PTH value at hypocalcemia in excess of $39.2 \mathrm{pg} / \mathrm{mL}$, with a mean PTH response to hypocalcemia of $20.1 \mathrm{pg} / \mathrm{mL}$.

The idea of a diminished parathyroid reserve, leading to a predisposition to hypocalcemia with stressors or illness, is supported by an examination of the clinical details surrounding hypocalcemic events. The majority (73\%) of hypocalcemic events in the subgroup presented in Table 1 had an acute stressor as a precipitating event, such as birth, operations, or illnesses. Others have also reported hypocalcemia in the postoperative state or during illness or stress.5,12,16,17

The concept of a diminished reserve, as opposed to a complete lack of function, is also supported by the fact that at random evaluations, intact PTH secretion generally falls within the normal range. As a whole, $81 \%$ of all intact PTH values in our study were within the normal range of $12-65 \mathrm{pg} / \mathrm{mL}$. Even in patients with a documented history of symptomatic hypocalcemia, 22 of 29 intact PTH values (76\%) were normal (illustrated in Fig. 2). Therefore, as in other forms of hypoparathyroidism, spot checks of intact PTH cannot be used as a screening tool; nor do they identify all patients at risk of hypocalcemic episodes. Our study did not examine whether patients were on any calcium or calcitriol supplementation at the time of the blood sampling, but calcitriol and calcium supplementation would, if they had any effect, suppress PTH levels.

Another conclusion from our study is that slightly more than half $(55 \%)$ of the patients with 22q11.2 deletion whose data we examined had a history of hypocalcemia. The initial presentation of hypocalcemia, in patients with 22q11.2 deletion and hypoparathyroidism, is usually early in life. Our study shows an incidence of $36 \%$ in the neonatal period, meaning that about two third of subjects who had a history of hypocalcemia had their first hypocalcemic event before 1 month of age. 
However, it is important to note that new onset hypocalcemia can occur several years after birth, as $8 \%$ of our patients had no history of hypocalcemia until a documented event occurred at 29 months of age or later. A previous study evaluating the incidence of hypocalcemia in 22q11.2 deletion syndrome reported an incidence of 58\% during infancy (before 2 years of age). ${ }^{18}$ Case reports have noted that some patients may not have their first episode of symptomatic hypocalcemia for decades. ${ }^{19}$ Our postneonatal incidence is similar to the $13-30 \%$ figure reported by Taylor et al. ${ }^{13}$ One confounder that needs to be kept in mind with regard to our results in neonates is the lack of ionized calcium results; in some cases, in a neonate, low total serum calcium may represent hypoalbuminemia, as opposed to true hypocalcemia.

Our study was limited by the retrospective design. Secondly, laboratory records before 1997 were not available via a computerized system, and patients born before this date were not included. As such, we are not able to comment on areas of interest such as the occurrence of hypocalcemia in adolescence or adulthood. The records on all patients at our center with this diagnosis born after 1997 were examined; however, simultaneous intact PTH and serum calcium values were available for only 33 of 103 patients. We report a prevalence of hypocalcemia of $55 \%$ in this population; this is a slightly lower percentage than has been reported elsewhere. Our lower number may stem from our status as a tertiary referral center, as patients may have had hypocalcemia before referral to our center. Sample size in terms of serum calcium and intact PTH was limited by our decision to only include those values for which both samples had been obtained simultaneously. We excluded intact PTH values drawn several hours after a total serum calcium value, as these results might be altered by a variety of factors, including treatment.

We note that these findings may be less applicable to those diagnosed with 22q11.2 deletion syndrome before the last decade, as it has become common over that period of time to perform fluorescence in situ hybridization testing on a far greater number of infants. Testing is now often done even if there is only one of the characteristic syndrome findings; therefore, the lower incidence of hypocalcemia could reflect "milder" infants being diagnosed. However, the study is still useful, as this cohort likely reflects a large fraction of those cared for by practitioners, and the majority of those cared for by pediatricians.

Our clinical practice is to check serum total calcium, or ionized calcium, along with intact PTH, in any infant aged $<3$ months suspected of, or known to have, 22q11.2 deletion syndrome. We screen calcium levels every 3-4 months through 1 year of age, as this is the period of time during which hypocalcemia is most likely to present. Thereafter, we screen annually, as occasionally hypocalcemia does occur without an identifiable precipitating stressor. Patients undergoing surgery have serum total calcium or ionized calcium screened preoperatively and postoperatively. Situations in which there is an identifiable stressor, severe enough to require hospitalization, or, in a young child, severe enough to cause significantly diminished oral intake, are periods of time during which attention to this issue is particularly necessary.

In subjects who have had hypocalcemia without a stressor, supplementation with calcium carbonate and calcitriol should be continued as needed, with dosage titrated in the manner typical for hypoparathyroidism. That is, we aim to maintain serum calcium toward the lower range of normal, or in a slightly subnormal range, with the aim of minimizing urinary calcium excretion. The course of action in subjects who are normocalcemic at baseline, but have experienced hypocalcemia with a stressor is less clear.

Prospective studies in this area are needed. Many patients require cardiac surgery and a period on cardiac bypass, a procedure that frequently results in a drop in serum calcium. A study with serial measurements of ionized calcium and intact PTH intraoperatively would serve the dual purpose of detecting hypocalcemia (ensuring patient safety), and defining intact PTH secretion at the time of hypocalcemia. These patients could then be followed up longitudinally, with periodic monitoring of total serum calcium, phosphorous, and intact PTH values. The incidence of nephrolithiasis and hypocalcemia with and without chronic calcium and/or calcitriol supplementation could then be examined. With most patients now diagnosed very early in life, and most patients observed at tertiary referral centers, such studies should be possible, with collaboration among cardiologists, endocrinologists, and geneticists.

In summary, hypoparathyroidism in 22q11.2 deletion syndrome is characterized by a phenomenon of diminished PTH reserve. Hypocalcemia can occur at baseline, and also manifests as an impaired ability to maintain normocalcemia with stressors. Random, baseline screens of calcium and intact PTH measurements are not sensitive screens for the presence of the $22 \mathrm{q} 11.2$ deletion, as most of such measurements will be within the normal range, even in children with a history of, and predisposition to, hypocalcemia. Intact PTH measurements at the time of hypocalcemia are also frequently within the normal range, but do not reach an appropriate elevation for the level of calcium.

\section{References}

1. Oskarsdottir S, Persson C, Eriksson BO, Fasth A. Incidence and prevalence of the 22q11 deletion syndrome: a population-based study in Western Sweden. Arch Dis Child 2004;89:148-151.

2. Ryan AK, Goodship JA, Wilson DI, Philip N, et al. Spectrum of clinical features associated with interstitial chromosome 22q11 deletions: a European collaborative study. J Med Genet 1997;34:798-804.

3. Botto LD, May K, Fernhoff PM, Correa A, et al. A population-based study of the 22q11.2 deletion: phenotype, incidence, and contribution to major birth defects in the population. Pediatrics 2003;112:101-107.

4. Lammer EJ, Opitz JM. The Digeorge anomaly as a developmental field defect. Am J Med Genet 1986;2:113-127.

5. Hieronimus S, Bec-Roche M, Pedeutour F, Lambert JC, et al. The spectrum of parathyroid gland dysfunction associated with the microdeletion 22q11. Eur J Endocrinol 2006; 155:47-52.

6. Digeorge AM. Discussion on a new concept of the cellular basis of immunity. J Pediatr 1965;37:389-394.

7. Conley ME, Beckwith JB, Mancer JF, Tenckhoff L. The spectrum of the Digeorge syndrome. J Pediatr 1979;94:883-890. 


\section{Kapadia et al.}

8. McDonald-McGinn DM, Goldmuntz E, Sullivan K, Eicher P, et al. The Philadelphia story: the 22q11.2 deletion: report on 250 patients. Genet Couns 1999; 10:11-24.

9. Weinzimer SA. Endocrine aspects of the 22q11 deletion syndrome. Genet Med 2001; 3:19-22.

10. Brauner R, Gonneville AL, Kindermans C, Bidois JL, et al. Parathyroid function and growth in 22q11 deletion syndrome. J Pediatr 2003;142:504-508.

11. Cuneo BF, Driscoll DA, Gidding SS, Langman CB. Evolution of latent hypoparathyroidism in familial 22q11 deletion syndrome. Am J Med Genet 1997;69:50-55.

12. Greig F, Paul E, DiMartino-Nardi J, Saenger P. Transient congenital hypoparathyroidism: resolution and recurrence in chromosome 22q11 deletion. J Pediatr 1996; 128:563-567.

13. Taylor SC, Morris G, Wilson D, Davies SJ, et al. Hypoparathyroidism and 22q11 deletion syndrome. Arch Dis Child 2003;88:520-522.
14. Al-Jenaidi F, Makitie O, Grunebaum E, Sochett E. Parathyroid gland dysfunction in 22q11.2 deletion syndrome. Horm Res 2007;67:117-122.

15. Garabedian M. Hypocalcemia and the chromosome 22q11 microdeletion. Genet Couns 1999;10:389-394.

16. Schaan BD, Huber J, Leite JC, Kiss A. Cardiac surgery unmasks latent hypoparathyroidism in a child with the 22q11.2 deletion syndrome. J Pediatr Endocrinol Metab 2006;19:943-946.

17. Cuneo BF, Langman CB, Ilbawi MN, Ramakrishnan V, et al. Latent Hypoparathyroidism in children with conotruncal cardiac defects. Circulation 1996;93:1702-1708.

18. Oskarsdottir S. Presenting phenotype in 100 children with the $22 \mathrm{q} 11$ deletion syndrome. Eur J Pediatr 2005;164:146-153.

19. Maaloof NM, Sakhaee K, Odvina CV. A case of chromosome 22q11 deletion syndrome diagnosed in a 32-year-old man with hypoparathyroidism. J Clin Endocrinol Metab 2004;89:4817-4820.

\section{Errata}

In the article "Genetics in clinical practice: general practitioners' educational priorities in European countries" which appeared in the February issue of Genetics In Medicine, the $P$ value on the first row of Table 4 (page 112) appeared as $<0.0000$ and should have appeared as $<0.0001$. This was due to an editing error. We regret any inconvenience this may have caused.

Julian-Reynier C, Nippert l, Calefato J-M, Harris H, et al. Genetics in clinical practice: general practitioners' educational priorities in European. Genet Med 2008;10:107-113.

In the article "The feasibility of online genetic testing for lung cancer susceptibility: uptake of a web-based protocol and decision outcomes" which appeared in the February issue of Genetics In Medicine on pages 121-130, incorrect data appeared in the "95\% CI" column of Table 2. This was due to an incorrect calculation. The number 1.67 should be replaced with $2.41,3.87$ replaced with 7.71, and 1.95 with 1.67. A correct table will appear online linked to this erratum.

O'Neill SC, White DB, Sanderson SC, Lipkus IM, et al. The feasibility of online genetic testing for lung cancer susceptibility: uptake of a web-based protocol and decision outcomes. Genet Med 2008;10:121-130. 